\title{
Spin-polarized transport properties in magnetic tunnel junctions with spin-orbit coupling
}

\author{
Y. Li ${ }^{\mathrm{a}, \mathrm{b}, *}$, C.R. Chang ${ }^{\mathrm{b}}$ \\ a Department of Materials Science and Engineering, School of Chemistry and Materials Engineering, Yunnan University, Kunming \\ 650091, PR China \\ ${ }^{\mathrm{b}}$ Department of Physics, National Taiwan University, Taipei 106, Taiwan
}

Received 20 June 2003; received in revised form 31 October 2003

\begin{abstract}
Effects on spin-polarized transport properties of interfacial scattering and random substitutional non-magnetic disorder in magnetic tunnel barrier are investigated numerically taking into account spin-orbit (SO) coupling and local $\mathrm{s}-\mathrm{d}$ exchange interaction under finite bias voltage. The method implemented with calculation of real space Green's function is employed in tight-binding model. Tunnel conductance is analyzed in terms of spin-conserving and spin-flip contributions due to mixing of spin states induced by the SO coupling. Spin polarization can even be inverted due to modification of spin-dependent density of states and shifted Fermi level with bias voltage. Interfacial spin-flip scattering plays a crucial role in the depression of spin-injection rate.
\end{abstract}

(C) 2003 Elsevier B.V. All rights reserved.

PACS: 73.40.Gk; 73.40.-c; 72.15.Gd

Keywords: Spin-polarized current injection; Magnetic tunnel junction; Spin-orbit coupling; Spin-flip scattering; Tunnel conductance and magnetoresistance

\section{Introduction}

Recent advances in spintronics have stimulated extensive study of spin-polarized transport in magnetic tunnel junctions (MTJs). The great interest in this field is due to numerous potential applications of spin injection [1,2], and efficient spin-polarized current injection is the key for various potential applications. Recent interest in

\footnotetext{
*Corresponding author. Department of Materials Science and Engineering, School of Chemistry and Materials Engineering, Yunnan University, Kunming 650091, PR China. Tel.: 86871-5031869; fax: 86-871-5033371.

E-mail address: lilucy@ynu.edu.cn (Y. Li).
}

spin injection into semiconductors $(\mathrm{S})$ is motivated by a desire to control over transport properties using gates voltage with additional spin degree of freedom in ferromagnet (FM), and a tunnel barrier $[3,4]$ was suggested to circumvent the conductivity mismatch between the FM and the S. It was shown theoretically that spin injection was improved considerably by a tunnel barrier [5], suggesting that tunneling may be a much more effective means for achieving efficient spin injection.

MTJs consist of two FM electrodes separated by an insulating (I) or a semiconductor barrier, and have been studied since 1970s [6]. Lots of efforts have been made to investigate the different factors affecting the spin polarization of tunnel current 
[7]. Though great progress has been achieved experimentally, one of the perplexing problems observed is the strong suppression of spin polarization with bias voltage [8]. The common wisdom about spin-polarized tunneling in MTJs is that tunnel conductance (TC) depends on the relative configuration of magnetizations in adjacent FMs, which implies that current carries with it information about the magnetization in the FM layers, and the spin-polarized transport depends strongly on the magnetic states at the FM-I(S) interfaces. The bias dependence characteristics could convey information about the detailed process of spinpolarized tunneling, and local magnetic fluctuations will be reflected in the shape and the amplitude of tunneling signal [9].

In this work, contributions of scattering potential, disorder and electric field to the spin-polarized transport properties in MTJs are studied numerically in the presence of spin-orbit (SO) coupling and local $\mathrm{s}-\mathrm{d}$ exchange interaction, which aims at revealing the roles of these spin-dependent interactions. The method presented earlier is employed in the tight-binding model [10]. The tunnel conductance is formulated in terms of real space Green's function, and calculated as the sum of spin-resolved contributions using recursive method.

\section{Theoretical treatment}

Taking into account SO coupling and local s-d exchange interaction, the second quantization expression for Hamiltonian is given by [10]

$$
\begin{aligned}
H= & \sum_{\mathbf{r}, \alpha, \beta}\left(\varepsilon_{\mathbf{r}} \delta_{\alpha \beta}+\frac{\gamma_{\mathbf{r}}}{2} \vec{\mu}_{\mathbf{r}} \cdot \vec{\sigma}_{\alpha \beta}\right) c_{\mathbf{r}, \alpha}^{+} c_{\mathbf{r}, \beta}+t \sum_{\left\langle\mathbf{r}, \mathbf{r}^{\prime}\right\rangle \alpha} c_{\mathbf{r}, \alpha}^{+} c_{\mathbf{r}^{\prime}, \alpha} \\
& +\sum_{\mathbf{r}, \alpha} \Phi_{\mathbf{r}} c_{\mathbf{r}, \alpha}^{+} c_{\mathbf{r}, \alpha}+H_{\mathrm{so}}, \\
H_{\text {so }}= & i \zeta_{\text {so }} \sum_{\mathbf{r}, \alpha, \beta}\left[\Delta \varepsilon_{\mathbf{r} \pm \mathbf{a}_{y}, \mathbf{r} \pm \mathbf{a}_{z}} c_{r, \alpha}^{+} c_{\mathbf{r} \pm \mathbf{a}_{y} \pm \mathbf{a}_{z}, \beta} \sigma_{\alpha \beta}^{x}\right. \\
& +\Delta \varepsilon_{\mathbf{r} \pm \mathbf{a}_{x}, \mathbf{r} \pm \mathbf{a}_{z}} c_{\mathbf{r}, \alpha}^{+} c_{\mathbf{r} \pm \mathbf{a}_{x} \pm \mathbf{a}_{z}, \beta} \sigma_{\alpha \beta}^{y} \\
& \left.+\Delta \varepsilon_{\mathbf{r} \pm \mathbf{a}_{x}, \mathbf{r} \pm \mathbf{a}_{z}} c_{\mathbf{r}, \alpha}^{+} c_{\mathbf{r} \pm \mathbf{a}_{x} \pm \mathbf{a}_{z}, \beta} \sigma_{\alpha \beta}^{z}\right] \\
t=- & \frac{\hbar^{2}}{2 m^{*} a^{2}},
\end{aligned}
$$

where $\mathbf{r}$ and $\alpha(\beta)$ are spatial and spin indices, respectively, $\gamma_{\mathbf{r}}$ the exchange splitting at magnetic site, $\delta_{\alpha \beta}$ the delta function, $\vec{\mu}_{\mathbf{r}}$ the unit vector in the direction of magnetization, $m^{*}$ and $\hbar$ the effective mass of electron and Plank constant, respectively. $c_{\mathbf{r}, \alpha}^{+}\left(c_{\mathbf{r}, \alpha}\right)$ is the creation (annihilation) operator with spin $\alpha$ at site $\mathbf{r}, \varepsilon_{\mathbf{r}}$ the on-site energy, and $\Delta \varepsilon_{\mathbf{r} \pm \mathbf{a}_{y}, \mathbf{r} \pm \mathbf{a}_{z}}=\varepsilon_{\mathbf{r} \pm \mathbf{a}_{y}}-\varepsilon_{\mathbf{r} \pm \mathbf{a}_{z}}, \quad \mathbf{r} \pm \mathbf{a}_{y}\left(\mathbf{a}_{z}\right)$ denote the nearest-neighbor sites from site $\mathbf{r}$ along $y(z)$-axis, $\mathbf{a}_{y}\left(\mathbf{a}_{z}\right)$ the vector paralleling to the $y(z)$ axis with length $a$, the lattice constant, $\zeta_{\text {so }}$ spin-orbit parameter, $\vec{\sigma}_{\alpha \beta}$ the Pauli operator with $\sigma_{\alpha \beta}^{x}, \sigma_{\alpha \beta}^{y}$, and $\sigma_{\alpha \beta}^{z}$ the matrix elements. $\Phi_{r}$ is the electrostatic potential with $\Phi_{r}=e V$ in the left and $\Phi_{r}=0$ in the right electrodes, respectively, assuming a linear potential drops across the barrier when small bias voltage $V$ is applied. The summation $\left\langle\mathbf{r}, \mathbf{r}^{\prime}\right\rangle$ runs over nearest-neighbor sites. All energies are measured in unit of nearest-neighbor hopping integral $|t|$, which is same for all pairs of nearest neighbors.

As shown in the above expression for $H_{\text {so }}$, spinflip scattering is expected due to mixing of spin states taking into account SO coupling in the presence of non-magnetic impurities [10].

The lattice Green's functions are defined in the tight-binding basis as [11]

$$
G_{\mathbf{n} \sigma, \mathbf{m} \sigma^{\prime}}^{ \pm}(i, j) \equiv\left\langle i, \mathbf{n}, \sigma\left|\frac{1}{E-H \pm \mathrm{i} \eta}\right| j, \mathbf{m}, \sigma^{\prime}\right\rangle,
$$

$G_{\mathbf{n} \sigma, \mathbf{m} \sigma^{\prime}}^{\mathrm{L} \pm}\left(i_{0}\right) \equiv\left\langle i_{0}, \mathbf{n}, \sigma\left|\frac{1}{E-H^{\mathrm{L}\left(i_{0}\right)} \pm \mathrm{i} \eta}\right| i_{0}, \mathbf{m}, \sigma^{\prime}\right\rangle$,

$|i, \mathbf{n}, \sigma\rangle \equiv c_{i, \mathbf{n}, \sigma}^{+}|0\rangle$,

in which $i, j$ represent the sites along current (yaxis) direction, $\mathbf{n}(\mathbf{m})$ is the vector in the transverse direction, $|0\rangle$ denotes vacuum state, $c_{i, \mathbf{n}, \sigma}^{+}$the creation operator with spin $\sigma$ at site $(\mathbf{i}, \mathbf{n})$, i $\eta$ an infinitesimal imaginary quantity, $H^{\mathrm{L}\left(i_{0}\right)}$ is the Hamiltonian for a system in which all sites $\mathbf{i} \succ \mathbf{i}_{\mathbf{0}}$ are deleted, $G^{+}\left(G^{-}\right)$denotes retarded (advanced) Green's function. We drop the spin indices and the \pm sign in the following derivation for simplicity.

Treating the nearest-neighbor hopping between two adjacent layers as a perturbation, Dyson's 
equation reads

$G=G^{0}+G^{0} T G$,

which leads to a set of equations that can be solved recursively. We obtain the recursion relations

$G^{\mathrm{L}}\left(i_{0}\right)=\left[G^{0}\left(i_{0}\right)^{-1}-T^{+} G^{\mathrm{L}}\left(i_{0}-1\right) T\right]^{-1}$,

$G^{\mathrm{L}}\left(i_{0}-1, i_{0}\right) \equiv-G^{\mathrm{L}}\left(i_{0}-1\right) T G^{\mathrm{L}}\left(i_{0}\right)$,

where $G^{0}\left(i_{0}\right)$ is the Green's function for the isolated layer at $\mathbf{i}=\mathbf{i}_{\mathbf{0}}, T$ the hopping matrix.

Initially, $G^{\mathrm{L}}(i)$ is calculated at the surface of semi-infinite left perfect lead, then one attaches the Green's function of a new layer to the already calculated propagator of its adjacent section at each step [11]. Starting at one end of the discretized structure, one proceeds through the entire sample by adding the next adjacent layer at each hopping location. After the last Green's function at the opposite end has been attached, the resulting total propagator between the two sides of the structure can be obtained:

$$
\begin{aligned}
G_{\mathrm{LR}}\left(1, N_{x}\right)= & G^{\mathrm{R}}\left(N_{x}\right) T\left(1-G^{\mathrm{L}}\left(N_{x}-1\right) \sum_{N_{x}-1}^{\mathrm{R}}\right)^{-1} \\
& \times G^{\mathrm{L}}\left(N_{x}-2, N_{x}-1\right),
\end{aligned}
$$

where $G^{\mathrm{R}}\left(N_{x}\right)$ is the surface Green's function for the semi-infinite right perfect lead, $\sum_{N_{x}-1}^{\mathrm{R}}=$ $T^{+} G^{\mathrm{R}}\left(N_{x}\right) T$ represents the self-energy of left lead plus the sample at site $N_{x}-1$ due to the hopping interaction onto the right lead.

Consider a MTJ consists of two semi-infinite FMs separated by an insulator (S) barrier $\left(2 \leqslant i \leqslant N_{y}-1\right)$, where $N_{y}$ is the site along the $y$ (current) direction. Along the transverse direction a periodical supercell with $N_{x} \times N_{z}$ size is assumed, where $N_{x}$ and $N_{z}$ are the sites along $x$ - and $z$-axis, respectively.

The calculation of conductance is based on the non-equilibrium Green's function formalism [12], which extended to spin systems in tight-binding model reads [10]

$$
G_{\alpha \beta}=\frac{e^{2}}{h} \operatorname{Tr}_{\mathbf{k}_{\|}}\left(\Gamma_{\mathrm{L}}^{\alpha} G_{\mathrm{LR} \alpha \beta}^{+}\left(1, N_{y}\right) \Gamma_{\mathrm{R}}^{\beta} G_{\mathrm{RL} \beta \alpha}^{-}\left(1, N_{y}\right)\right),
$$

it formulates the transport properties in terms of transmission function, $\operatorname{Tr}_{\mathbf{k}_{\|}}\left(\Gamma_{\mathrm{L}}^{\alpha} G_{\mathrm{LR} \alpha \beta}^{+}\left(1, N_{y}\right) \Gamma_{\mathrm{R}}^{\beta}\right.$ $\left.G_{\mathrm{RL} \beta \alpha}^{-}\left(1, N_{y}\right)\right)$, in which $\operatorname{Tr}$ represents the trace for $2 N_{x} N_{z} \times 2 N_{x} N_{z}$ matrix, and $\mathbf{k}_{\|}$is the wave vector in the two-dimensional Brillouin zone corresponding to the supercell. L (R) refers to the left (right) FM electrode, and

$\Gamma_{\mathrm{L}}^{\alpha} \equiv \mathrm{i} t^{2}\left[G_{\mathrm{L} \alpha}^{+}(1)-G_{\mathrm{L} \alpha}^{-}(1)\right]$,

$\Gamma_{\mathrm{R}}^{\beta} \equiv \mathrm{i} t^{2}\left[G_{\mathrm{R} \beta}^{+}\left(N_{y}\right)-G_{\mathrm{R} \beta}^{-}\left(N_{y}\right)\right]$,

which describe coupling at the interfaces, where $G_{\mathrm{L} \beta}^{ \pm}(1)\left(G_{\mathrm{R} \beta}^{ \pm}\left(N_{y}\right)\right)$ is the surface Green's function for the unconnected left (right) FM electrode. The interlayer Green's functions $G_{\mathrm{LR} \alpha \beta}^{ \pm}\left(1, N_{y}\right)$ and $G_{\mathrm{RL} \beta \alpha}^{ \pm}\left(1, N_{y}\right)$ describing the dynamics of electrons are calculated using the above recursive relations.

We get the spin-resolved total tunnel conductance which is consists of spin-conserving and spin-flip parts due to mixing of spin states caused by $\mathrm{SO}$ coupling [10],

$G=G_{\mathrm{sc}}+G_{\mathrm{sf}}$,

$G_{\mathrm{sc}}=G_{\uparrow \uparrow}+G_{\downarrow \downarrow}$,

$G_{\mathrm{sf}}=G_{\uparrow \downarrow}+G_{\downarrow \uparrow}$,

where $\uparrow \uparrow(\downarrow \downarrow)$ indicates that the magnetic moment of electron is parallel (P) (anti-parallel (AP)) to the magnetization direction in the left and right $\mathrm{FM}$ electrodes, respectively.

\section{Results and conclusion}

To evaluate the influence of spin-flip scattering caused by SO coupling, non-magnetic disorder simulated by random substitutional on-site energy in the barrier is considered under finite bias voltage. We apply the above theory to a MTJ composed of two adjacent Co electrodes with Fermi energy $E_{\mathrm{F}}$ and exchange splitting $\gamma_{\mathrm{r}}$ taken to be $-5.1|t|$ and $1.0|t|$, respectively [13]. The spin independent on-site energy for FM electrodes is taken to be $\varepsilon_{\mathbf{r}}=0$, and inside the barrier $(2 \leqslant i \leqslant 5) \varepsilon_{\mathbf{r}}$ is set to be the barrier height $U=$ $2.0|t|$ except at the impurities sites, where $\varepsilon_{\mathbf{r}}=3.0|t|$ with concentration $c_{i}=0.1$, which is reasonable to mimic practical situations. We consider different cases, i.e. tunneling through uniform and disordered barrier with and without SO coupling, respectively. The calculations are performed for 
$10 \times 10$ lateral supercell at zero-temperature limit, and applied bias voltage ranges from $-0.5|t|$ to $1.0|t|$. For numerical calculation, $t=-1$ is assumed, and single-orbit basis is used for simplicity.

Fig. 1 shows the dependence of spin-flip ratio, $G_{\text {sf }} / G$, on the thickness of disordered barrier, which is sensitive to the potential profile in the barrier since spin-flip processes induced by SO interaction proportion to the local on-site energy difference (see (1b)). We find that the spin-flip scattering is much stronger for AP alignment of magnetizations in adjacent FMs than for $P$ alignment. The reason is the reversal of spin dependence of initial and final density of states (DOS) in the spin-flip processes. As is well known, in the spin-conserving tunneling, TC $\left(G_{\mathrm{sc}}\right)$ increases when the magnetization direction of the adjacent FMs changes from anti-parallel to parallel, which originates from the difference between the densities of majority and minority spin states at the Fermi level in the adjacent FM electrodes. When the role of majority and minority spin DOS is reversed for electrons undergoing spin-flip scattering, reverted result would be expected, i.e. $G_{\mathrm{sf}}$ is larger for AP than for $\mathrm{P}$ configuration.

In Fig. 2, TC of junctions with and without disorder in the barrier is plotted as a function of bias voltage for $\mathrm{P}$ and AP configurations,

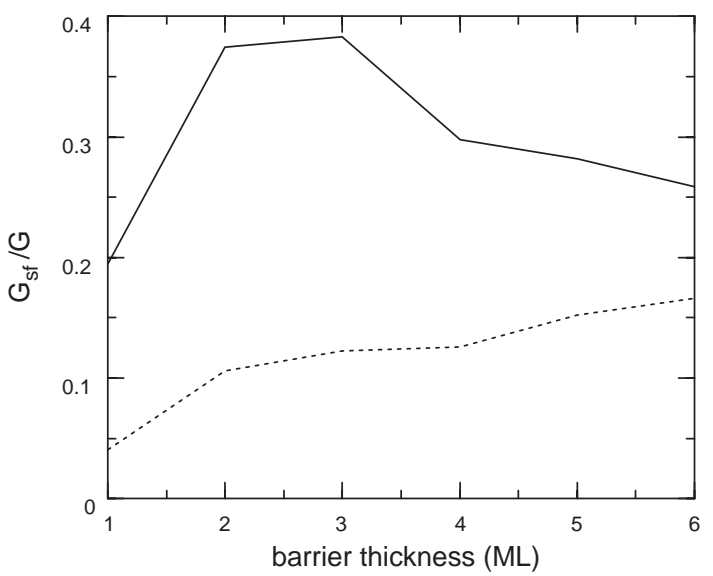

Fig. 1. The ratio, $G_{\text {sf }} / G$ versus barrier thickness for $\mathrm{P}$ (dashed line) and AP (solid line) configurations, respectively, for $\zeta_{\text {so }}=0.3$. The parameters used: $E_{\mathrm{F}}=-5.1|t|, \gamma_{r}=1.0|t|, U=$ $2.0|t|, \varepsilon_{r}=3.0|t|, c_{i}=0.1$.

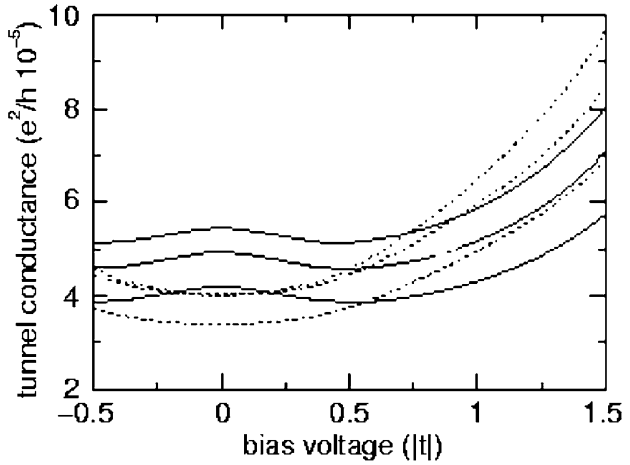

Fig. 2. Bias voltage dependence of TC. P configuration (solid line): with disorder in the barrier, $\zeta_{\text {so }}=0$ (upper branch), $\zeta_{\text {so }}=$ 0.15 (middle branch); uniform barrier, $\zeta_{\text {so }}=0.15$ (lower branch). AP configuration (dotted line): with disorder in the barrier, $\zeta_{\text {so }}=0$ (upper branch), $\zeta_{\text {so }}=0.15$ (middle branch); uniform barrier, $\zeta_{\mathrm{so}}=0.15$ (lower branch). The parameters used are the same as in Fig. 1.

respectively. We find that the $\mathrm{TC}$ increases while hopping through the impurity states in the barrier for both $\mathrm{P}$ and AP alignments (the upper branches of the solid and dotted lines, respectively, see Fig. 2), but decreases by the spin-flip scattering when SO coupling is considered (the middle branches of the solid and dotted lines, respectively, see Fig. 2). As can be seen from the TC curves in Fig. 2, for the $\mathrm{P}$ alignment the TC decreases at small bias, then increases gradually at almost the same slope for different cases considered in the calculations after reaching the minimum value at certain voltage, but for AP alignment a monotonous increases with bias voltage are shown, and at steeper slope after the crossover with the TC curves in the $\mathrm{P}$ configuration. The conductance peaks, i.e. the so-called zero-bias anomaly, exhibits for $\mathrm{P}$ configuration, which may reflect the specific DOS at the Fermi level as described by Wyatt for tunneling through an insulator barrier [14]. From the $G-V$ characteristics we come to the conclusion that the bias voltage dependence of TC is determined by local $\mathrm{s}-\mathrm{d}$ exchange interaction between spin-polarized current and local magnetic states. Symmetric results would be expected about the zero-bias due to the symmetric tunnel junction structure considered. The current-bias curves are shown in Fig. 3, similar characteristic of bias 


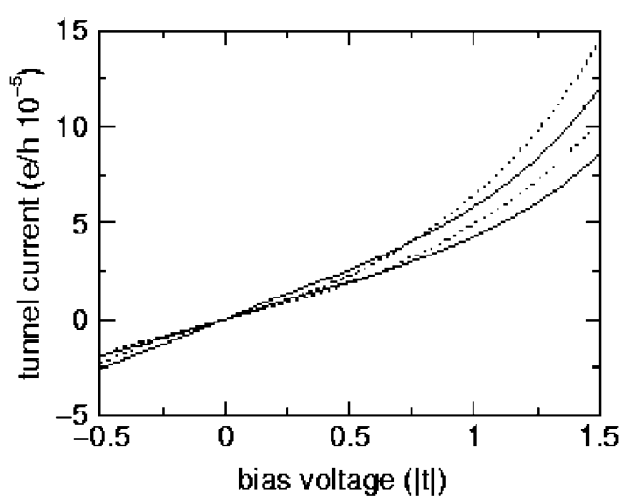

Fig. 3. Bias voltage dependence of tunnel current. P configuration (solid line): with disorder in the barrier, $\zeta_{\text {so }}=0$ (upper branch); uniform barrier, $\zeta_{\text {so }}=0.15$ (lower branch). AP configuration (dotted line): with disorder in the barrier, $\zeta_{\mathrm{so}}=$ 0 (upper branch); uniform barrier, $\zeta_{\text {so }}=0.15$ (lower branch). The parameters used are the same as in Fig. 1.

voltage dependence is obtained. We get stronger current for $\mathrm{P}$ alignment at small bias, but crossover occurs as the bias voltage increases, which may reflect the bias dependence of DOS.

Bias voltage dependence of TMR ratio, defined as $T M R=\Delta G / \bar{G}=\left(G_{\mathrm{p}}-G_{\mathrm{ap}}\right) / \bar{G}[15]$, is shown in Fig. 4, where $G_{\mathrm{p}}\left(G_{\mathrm{ap}}\right)$ is the total TC for $\mathrm{P}(\mathrm{AP})$ alignment of magnetizations in the adjacent FM electrodes, $\bar{G}$ is the average value of $G_{\mathrm{p}}$ and $G_{\text {ap }}$, the difference $\Delta G$ can be a measure for spininjection, a standard although indirect way to detect spin-injection experimentally. As explained above, the spin-conserving tunneling gives a positive TMR, while the spin-flip processes contribute to a negative effect, thus the total TC, which is the sum of the two contributions will be decreased in the presence of SO coupling. We find that non-magnetic disorder in the barrier does not has much effects on TMR ratio, but spin-flip interfacial scattering plays a decisive role in the depression of TMR ratio, and thus the spin injection coefficient is reduced significantly. The TMR decreases due to the spin-flip scattering caused by SO coupling by almost the same order of magnitude for the different cases considered. Since a scattering potential always exists at the FM-I(S) interfaces, our results may suggest that strong scattering potential should be avoided to minimum such unfavorable influence for efficient

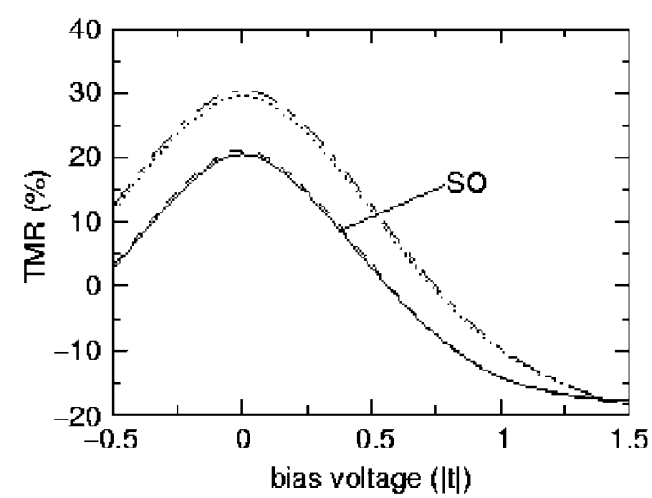

Fig. 4. TMR as a function of applied bias voltage. $\zeta_{\text {so }}=0$ : uniform barrier (dot-dashed line); with disorder in the barrier (dotted line). $\zeta_{\text {so }}=0.15$ : uniform barrier (dashed line); with disorder in the barrier (solid line). The parameters used are the same as in Fig. 1.

spin-injection. The sign of spin-polarization changes at a threshed bias due to shifted Fermi level and modification of the spin dependent DOS with bias voltage. Bias dependence of DOS was proposed recently to explain the rapid decrease of resistance and TMR in magnetic tunnel junctions [16].

In summary, the transport properties of $\mathrm{Co}|\mathrm{I}(\mathrm{S})| \mathrm{Co}$ MTJs are investigated numerically in the presence of scattering potential and nonmagnetic disorder in linear response region when finite bias voltage is applied. The calculations are performed in single-orbit tight-binding model taking into account SO coupling and local s-d exchange interaction. We find that spin-flip scattering (SFS) induced by SO coupling contributes to a negative magnetoresistance, and the interfacial SFS plays a critical role in the reduction of spin-injection rate. Spin polarization can even be inverted due to modification of spin dependent DOS and the shifted Fermi level with bias voltage.

\section{References}

[1] G.A. Prinz, Science 282 (1998) 1660.

[2] S.A. Wolf, D.D. Awschalom, R.A. Buhrman, J.M. Daughton, S.von. Molnar, M.L. Roukes, A.Y. Chtchelkanova, D.M. Treger, Science 294 (2001) 1488. 
[3] E.I. Rashba, Phys. Rev. B 62 (2000) R16267.

[4] A. Fert, H. Jaffrès, Phys. Rev. B 64 (2001) 184420.

[5] H.B. Heersche, Th. Schäpers, J. Nitta, H. Takayanagi, Phys. Rev. B 64 (2001) 161307.

[6] P.M. Tedrow, R. Meservey, Phys. Rev. B 37 (1973) 318; M. Julliere, Phys. Lett. 54 (1975) 225.

[7] S. Zhang, P.M. Levy, Phys. Rev. Lett. 79 (1997) 3744; J. S. Moodera, G. Mathon, J. Magn. Magn. Mater. 200 (1999) 248; J.M. De Teresa, A. Barthélémy, A. Fert, J. P. Contour, f. Montaigne, P. Seneor, Science 286 (1999) 507; D. Bagrets, A. Bagrets, A. Vedyayev, B. Dieny, Phys. Rev. B 65 (2002) 064430.

[8] J.S. Moodera, L.R. Kinder, T.M. Wong, R. Meservey, Phys. Rev. Lett. 74 (1995) 327.
[9] C. Heide, A.I. Krikunov, Yu.F. Ogrin, P.E. Zilberman, J. Appl. Phys. 87 (2000) 5221.

[10] Y. Li, C.R. Chang, Phys. Lett. A 287 (2001) 415.

[11] D.K. Ferry, S.M. Goodnick, Transport in Nanostructures, Cambridge University Press, Cambridge, 1997.

[12] S. Datta, J. Phys. Condens. Matter 2 (1990) 8023.

[13] V.L. Moruzzi, J.F. Janak, A.R. Williams, Calculated Electronic Properties of Metals, Pergamon, Oxford, 1978.

[14] A.F.G. Wyatt, Phys. Rev. Lett. 13 (1964) 401.

[15] S. Maekawa, U. Gäfvert, IEEE Trans. Magn. 18 (1982) 707.

[16] X.H. Xiang, T. Zhu, J. Du, G. Landry, J.Q. Xiao, Phys. Rev. B 66 (2002) 174407. 